\title{
Cooperation in an Adaptive Network
}

\author{
M.G. Zimmermann ${ }^{1,2}$, V.M. Eguíluz ${ }^{1,3}$, M. San Miguel $^{1}$, A. \\ Spadaro $^{4}$
}

\begin{abstract}
We study the dynamics of a set of agents distributed in the nodes of an adaptive network. Each agent plays with all its neighbors a weak prisoner's dilemma collecting a total payoff. We study the case where the network adapts locally depending on the total payoff of the agents. In the parameter regime considered, a steady state is always reached (strategies and network configuration remain stationary), where co-operation is highly enhanced. However, when the adaptability of the network and the incentive for defection are high enough, we show that a slight perturbation of the steady state induces large oscillations (with cascades) in behavior between the nearly all-defectors state and the all-cooperators outcome.
\end{abstract}

Keywords: Social organisation, Networks, Game theory, Agent based models, Weak Prisoner's Dilemma

\footnotetext{
${ }^{1}$ Instituto Mediterráneo de Estudios Avanzados IMEDEA(CSIC-UIB), E07071 Palma de Mallorca, Spain. Supplementary information at http:www.imedea.uib.es.

${ }^{2}$ Present address: Departamento de Física, Universidad de Buenos Aires, Pabellón I Ciudad Universitaria, 1428 Buenos Aires, Argentina.

${ }^{3}$ Corresponding author email: victor@delta.ens.fr. Present address: DELTA (Joint Research Unit CNRS-ENS-EHESS), 48 Bd Jourdan, 75014 Paris, France.

${ }^{4}$ DELTA (Joint research unit CNRS-ENS-EHESS) and Department of Economics and Business, Universitat de les Illes Balears, E07071 Palma de Mallorca, Spain.
} 
Résumé : Nous étudions la dynamique d'un groupe d'agents distribués dans
les noeuds d'un réseau. Chaque agent joue avec tous ses voisins un Faible Di-
lemme du Prisonnier en collectant un pay-off total. Nous étudions le cas d'un
réseau qui s'adapte localement selon les pay-off des agents. Premièrement, un
état stationnaire est toujours trouvé pour les paramètres étudiés, où la frac-
tion d'agents qui coopère est stationnaire. Pour quelques paramètres même
on a trouvé un état presque complètement coopérative. Cependant, quand
l'adaptabilité du réseau et l'incitation á la défection sont assez grands, on
montre que une petite perturbation de l'état stationnaire induit des grandes
oscillations (avec cascades) dans le comportement entre l état quasi-tout-
defection et quasi-tout-cooperation.

Mots-clés : Organisation sociale, Réseaux, Théorie des jeux, Models d'agents, Faible Dilemme du Prisonnier

\section{Introduction}

A subject which has intrigued many economists is how the organization of an economy arises and evolves. Agents interact in multiple ways, as for example information transmission in financial markets ${ }^{5}$, or firms competition or collusion in an oligopoly ${ }^{6}$. These interactions can be described by a set economic agents which sit in the nodes of a network. Among other approaches (for a review see [KIR 99]), the mechanisms of interactions and the emergence of collaboration in a group of agents have been analyzed by the use of evolutionary game theory [WEI 96]. Using the Prisoner's Dilemma (PD) game, [AXE 81] and [AXE 84] showed how cooperation may be sustained by a population of agents meeting repeatedly and having certain degree of rationality. Strategies were allowed to mutate and reproduce in proportion to the difference between the agent's payoff and the population's average payoff. Cooperation was shown to be sustained by the use of the evolutionary stable strategy Tit-For-Tat. This approach assumes that the game is carried out by randomly matching a pair of agents from a fixed population. This assumption seems plausible for systems with a large number of agents where the probability of playing several times with the same agent is extremely low, and for systems where the agents cannot create links or preferences between them.

However, in many social and economic environments this assumption does not hold, and each agent interacts only with a small subset of the whole population $^{7}$. One reason for this might be that agents have imperfect information on the whole population, except for a small subset which can be considered as "the neighbors".

\footnotetext{
${ }^{5}$ See for example [BAN 92], [KIR 93], [CON 99], [EGU 99].

${ }^{6}$ See for example [FER 98], [GOY 99].

${ }^{7}$ For a deep study of the dynamics of a PD game with different strategies, evolution of the strategies and networks see [COH 99].
} 
In this paper we introduce a spatial Weak Prisoner's Dilemma (WPD) ${ }^{8}$ model played on an endogenously adaptive network where cooperation is promoted and sustained by local interactions and the adaptation of the network. To simplify matters we do not allow the strategies to evolve and consider only zero-memory strategies, i.e. all-C [all-D] strategy means doing $\mathrm{C}[\mathrm{D}]$ all the time irrespective of the outcome.

Each agent plays the same strategy either $\mathrm{C}$ (cooperate) or D (defect) with all its local neighbors, as in [NOW 92]. Each agent revises his strategy at each iteration of the game and imitates the strategy of the neighbor with highest aggregate payoff. Finally we consider the adaptation of the network by allowing the possibility of changing the neighborhood whenever an agent was unsatisfied and imitated its best local neighbor. Specifically, if this best local neighbor is a D-neighbor, the imitating agent cuts the link with the D-neighbor, with some probability, and establishes a new link selecting randomly a new agent to become its neighbor from the whole population of agents.

As possible scenarios where these cooperative networks might arise we can mention a network of individuals or firms that have some agreements among them but with some risk involved in the cooperation. The agreements correspond to the links in the network, and respecting their agreements results in playing Cooperation (C), while not respecting their agreement corresponds to Defection (D). An interesting application might be a network of firms which share their research and development outcome. Another application might be a network of scientist which agree to collaborate in different projects.

We have determined from numerical experiments the following main results. First, for the range of parameters studied, the network always reaches a steady state where the fraction of cooperating agents (C-agents) is high. In these states the network remains stationary. However, most of the agents are unsatisfied and thus are continuously imitating their best neighbor's strategy, which is the same strategy they are using. This does not change the local payoffs and thus it remains in a stationary state. We find also that although the dynamics always converges to a steady state, when the incentive to defect is sufficiently high, a perturbation may induce large oscillations in the fraction of $\mathrm{C}$-agents together with a large reorganization of the network. These oscillations stretch between the quasi-all C-agents, to the quasi-all D-agents networks. We stress the fact that these are transient and they last for some time before a new steady state is reached. In most cases high cooperation is again reached, but there is a small probability to reach the quasi-all D-network. Thus we show that although cooperation is greatly enhanced by such a network update, the system may organize in a state where an exogenous or stochastic perturbation

\footnotetext{
${ }^{8}$ We will explain in the next section the difference between the classical Prisoner's Dilemma and the WPD we use in this paper.
} 
may produce drastic changes on a finite time. The oscillations can be triggered by a change of strategy of a single agent with a large number of links. This identifies the importance of the highly-connected agents which play a leadership role in the collective dynamics of the system. Finally, it is interesting to study the characteristics of the network that emerges from the interaction between agents. Such structure is far from trivial in the sense that presents a given pattern.

The paper is organized as follows. The next section defines the adaptive model. Section 3 deals with the conducted numerical experiments. First the case of a fixed network is revisited, and then the full adaptive network is presented and discussed. Finally, in Section 4, we discuss our results and open problems.

\section{The model}

We consider an adaptive game where $N$ agents play a WPD game on a network $\Gamma$. Each agent is located in a node of the network (and there is a single agent per node). Two agents are neighbors if they are directly connected by one link. We define the neighborhood of agent $i$ as the subset of $\Gamma$ which are neighbors of $i$, and we represent it as neigh $(i)$; its cardinal is $k_{i}$. Each agent plays only with those other agents connected by one $l i n k^{9}$. If $N_{l}$ is the total number of links and $k_{i}$ is the number of links of node $i$, then the average connectivity of a network, $k$, is defined as the average number of links per node

$$
k=\frac{\sum_{i=1}^{N} k_{i}}{N}=\frac{2 N_{l}}{N} .
$$

In this paper we consider two different kind of initial networks on which the agents start the game: regular lattices in two-dimensions and random networks. A two-dimensional regular lattice correspond to a Manhattan-like grid, where the nodes are the intersections of streets and avenues. We will consider firstneighbor interaction that corresponds to moving North, South, West and East (thus $k_{i}=4, \forall i$ and $k=4$ ), while the 2nd-neighbor interaction corresponds to the possible moves a King in Chess can make (thus $k_{i}=8, \forall i$ and $\left.k=8\right)^{10}$. Random networks of average connectivity $k$ are formed by distributing $N_{l}=$ $k N / 2$ bidirectional links between pairs of nodes $(i, j)$, with the constraint that $(i, j)=(j, i)$ (bidirectional links). The resulting distribution of the number of links in the network is Gaussian with the maximum located at the average connectivity $k$.

\footnotetext{
${ }^{9} \mathrm{We}$ assume that the links are bidirectional. More general situations can be considered with uni-directional links, but we do not explore this further.

${ }^{10}$ Previous results of PD game in regular lattices are investigated in for example [NOW 92].
} 
Let us denote by $s_{i}(t)=\{0,1\}$ the strategy of agent $i$ at time step $t$, where $s_{i}=1$ corresponds to play cooperation (C), and $s_{i}=0$ corresponds to defection (D). These will be referred to as $\mathrm{C}$-agents or $\mathrm{D}$-agents, respectively. The payoff matrix for a 2-agents game is:

\begin{tabular}{l|l|l|} 
& $\mathrm{C}$ & $\mathrm{D}$ \\
\hline $\mathrm{C}$ & $\sigma, \sigma$ & $0, b$ \\
\hline $\mathrm{D}$ & $b, 0$ & $\delta, \delta$ \\
\hline
\end{tabular}

where we take $b>\sigma>\delta>0$, and $b / 2<\sigma$ for a Prisoner's Dilemma game. In this paper we study the case of a Weak Prisoner's Dilemma (WPD): a Prisoner's Dilemma is Weak when $\delta=0$. In a standard Prisoner's Dilemma there exists a unique Nash equilibrium $(\mathrm{D}, \mathrm{D})$ while in WPD either a $(\mathrm{C}, \mathrm{D}),(\mathrm{D}, \mathrm{C})$ or $(\mathrm{D}, \mathrm{D})$ may be attained as a Nash equilibrium.

We use in this Paper the WPD for which the analysis is much simpler, taking into account that [NOW 92, LIN 94] showed that, at least for a fixed regular network, the results do not change qualitatively when using $1 \gg \delta>0$.

We consider the situation in which agents always tries to maximize their utility, and therefore seeks the largest possible benefit from their local interactions in the network $\Gamma$. We assume each agent plays the same strategy with all its neighbors neigh $(i)$ and only with them. The game is played synchronously, i.e. the players decide their strategy in advance and they all play at the same time. The strategy update of agent $i$ is as follows:

1. Each agent $i$ plays the WPD game with each neighbor using the same strategy $s_{i}$ and collecting a total individual payoff $\Pi_{i}$.

2. Agent $i$ revises its current strategy at each iteration of the game (i.e., at every time step), and updates it by imitating the strategy of its neighbor with a highest pay-off. Agent $i$ is said to be satisfied if his pay-off is the maximum of its neighbors; otherwise it will be unsatisfied and it will revise its strategy.

3. The agents have also the possibility of an extra action which adapts its neighborhood. Namely we consider:

Network Rule: if agent $i$ is unsatisfied and imitates from a D-agent $j$, then with probability $p, i$ breaks the link with $j$ and establishes a new link with another agent chosen randomly in the network $\Gamma$.

This rule leads to a time evolution of the local connectivity of the network, leaving the global connectivity $k$, as defined in Eq. 1, constant. For each agent $i$ which imitates a D-agent $j$ and decides to break the link and choose a new 
agent $\beta$, neigh $(i)$ changes by replacing $j \rightarrow \beta$ and $k_{i}(t+1)=k_{i}(t)$. However $j$ will lose a link, $k_{j}(t+1)=k_{j}(t)-1$, and the new agent $\beta$ will increase its local connectivity $k_{\beta}(t+1)=k_{\beta}(t)+1$. Thus, the network adaptation introduces a diversity in the agents local neighborhoods.

Agreements between satisfied agents do not change. This does not mean that new agreements with other agents are not possible (these agents may always receive new links), but those which exist remain untouched. The same is true for unsatisfied $\mathrm{C}$-agents imitating another $\mathrm{C}$-agent. However neighborhoods of $\mathrm{D}$-agents that have the maximum pay-off in the neighborhood could change abruptly in just one iteration.

The network rule can be understood as a risk minimization. If agent $i$ is a D-agent and is unsatisfied he minimizes the risk of cooperation by taking chances and selecting a new neighbor from the whole network. In the context of game theory, this can be seen as a retaliation, because is highly unlikely that he will play with those agents in the future.

The probability $p$ represents a transaction cost of breaking one agreement and establishing a new agreement with a new partner. It can also be understood as a measure of the tolerance of being exploited. We would like to stress that the transaction cost has two components: first, the cost of breaking an agreement and second, the cost of finding a partner and that this new partner accepts the agreement ${ }^{11}$. The case $p=0$ corresponds to an infinite transaction cost for breaking the link, while $p=1$ corresponds to the limiting case of no transaction cost. It is clear that breaking at the same time more than one link, and finding their respective partners is more unlikely. The probability $p$ is also a measure of the adaptability of the network to the results of the game at each iteration.

It will be useful to define the looking function of agent $i$ which will be denoted by $l(i)$. This function points to $i$ 's neighbor with highest payoff, including himself; thus if $i$ is not imitating any neighbor then $l(i)=i$. From this we can define an agent being a local maximum in payoff as the one which satisfies $i=l(i)$ and at least one of its neighbors is looking at it.

Suppose that at a given time step there is a D-agent which is a local maximum. This implies that at the next time step, the D-strategy will be replicated on all of its neighbors, and its links will be destroyed with probability $p$. Thus, a D-local maximum at one time step, is an unstable situation where the agent looses a fraction $p$ of all its links on the next time step, and these links will be replaced by new neighbors.

With the network rule implemented in this Paper the total number of links

\footnotetext{
${ }^{11}$ One could separate these two costs, and would have a process of breaking links (with a given probability $q$ ) and another process of generation of links (with a probability $r$ ). This line of research is not explored in this paper.
} 


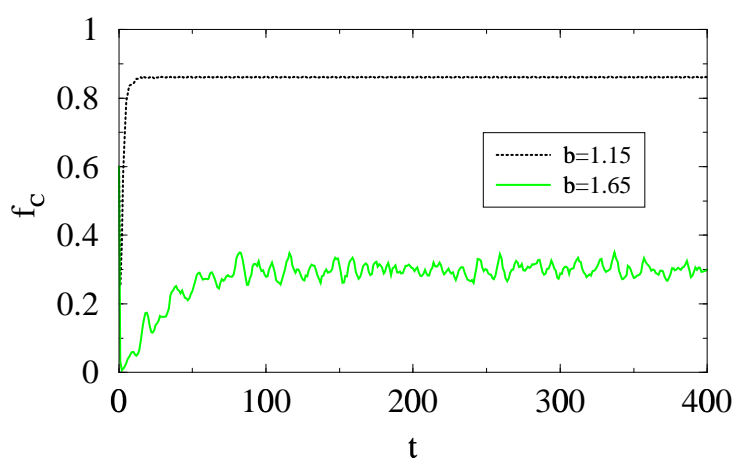

Figure 1: Time evolution of the fraction of C-agents $f_{C}$ for a non-adaptive network $p=0$, in a two-dimensional regular lattice with second-neighbors interaction ( $k=8$ : (top trajectory) asymptotic periodic trajectory for $b=1.15$ and (bottom trajectory) chaotic trajectory for $b=1.65$. Payoff matrix: $\sigma=1$, $\delta=0$.

in the network $\Gamma$ is conserved. We do not take into account more complicated network dynamics as spontaneous creation or destruction of links, that will break the conservation of total number of links.

\section{Numerical studies}

We have characterized numerically the model described above using as parameter the incentive to defect $b$. We have used as initial networks random networks with an average connectivity $k=4$ and $k=8$ and two-dimensional regular lattices with first- $(k=4)$ and second-neighbor $(k=8)$ interaction. We have also fixed the adaptability $p=1$. The statistical measures that we have studied are:

- The fraction (normalized to the whole population $N$ ) of cooperating agents (C-agents), denoted by $f_{C}=\left(\sum_{i=1}^{N} s_{i}\right) / N$.

- The average payoff per agent $\Pi=\left(\sum_{i=1}^{N} \Pi_{i}\right) / N$ of the whole network.

- The probability of having a link between two C-agents, $p_{C C}$, between a $\mathrm{C}$-agent and a $\mathrm{D}$-agent, $p_{C D}$, and between two D-agents, $p_{D D}$. These probabilities satisfy:

$$
1=p_{C C}+2 p_{C D}+p_{D D}
$$




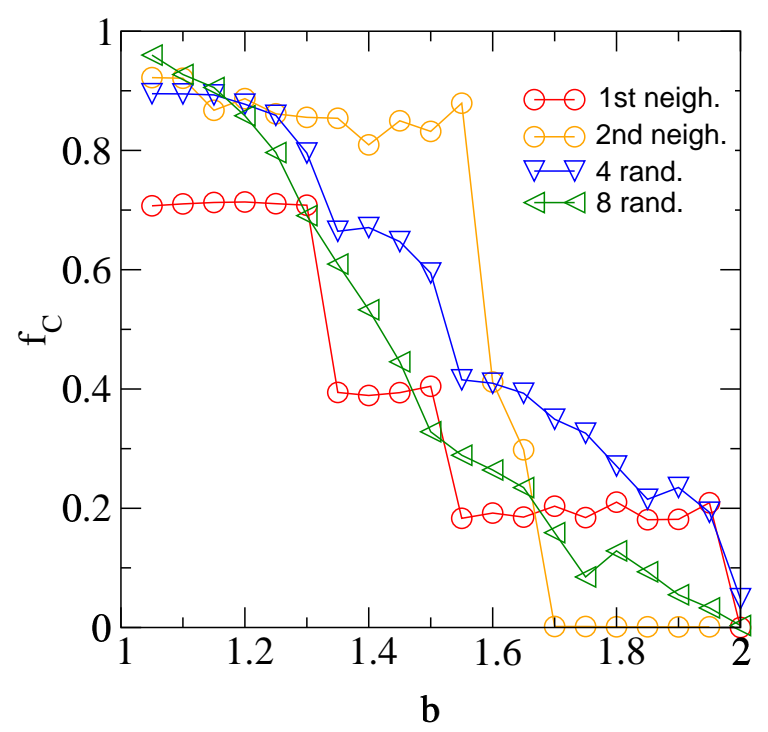

Figure 2: No network adaptation $(p=0)$. Average fraction of C-agents, $f_{C}$, vs. defecting incentive, $b$, in 1st and 2nd neighbors regular lattices, and in random networks with $k=\{4,8\}$.

In the numerical simulations we maintain fixed the parameters $\delta=0$ and $\sigma=1$ of the payoff matrix and we vary $b$ in the range $1<b<2$. Finally, in most simulations we take $N=10000$ agents, and we start with an initial population of $0.6 \mathrm{~N}$ C-agents randomly distributed in the network $\Gamma$.

The game in a fixed network $(p=0)$ and regular lattices, has been previously studied by [NOW 94, NOW 92, NOW 93]. Typically the behavior of the fraction of $\mathrm{C}$-agents, $f_{C}$, can show different features. The simplest is an asymptotic stationary or periodic state, where $f_{C}$ remains stationary or fluctuates periodically. A more complex behavior is the spatio-temporal chaotic regime, where $f_{C}$ fluctuates in time around an average value while the spatial distribution of $\mathrm{C}$ - and $\mathrm{D}$ - agents present evolving patterns at each iteration. Finally, if the incentive to defect $b$, is high enough the asymptotic state of the system is all D-agents. Also the introduction of elements that disrupt the spatial correlations present when the game is played in a regular lattices (e.g., random lattices, noise and errors in the imitating process), was shown to destroy the periodic fluctuations and regular patterns observed, as expected.

One of the main result of these studies is that partial cooperation can be sustained by local interactions, together with a very simple choice of strategies which does not include memory. This can be illustrated by studying the average fraction of $\mathrm{C}$-agents for an increasing value of the incentive to defect $b$ (see 


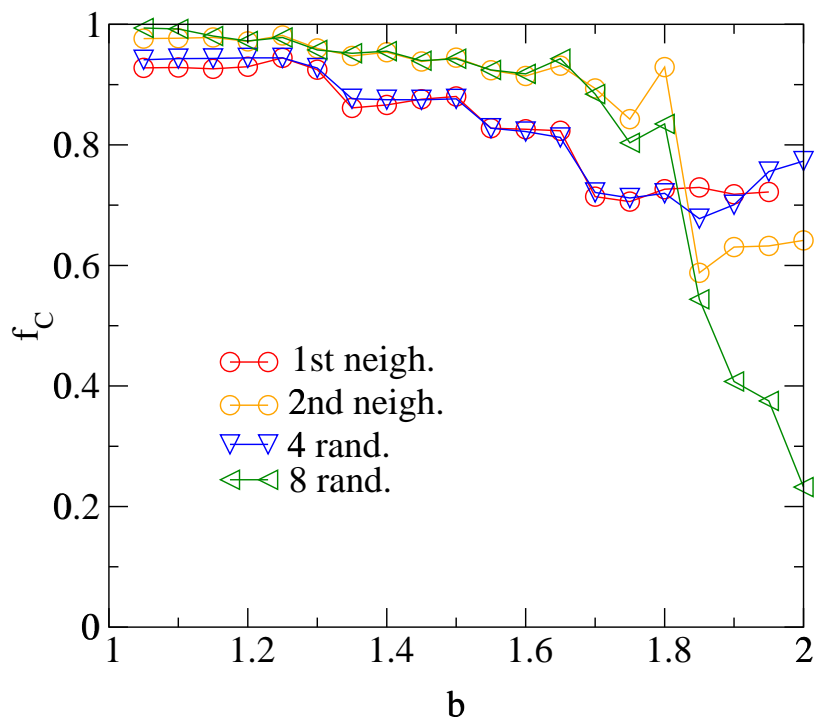

Figure 3: Network adaptation $(p=1)$. Fraction of cooperative agents vs. $b$ in different initial networks.

Fig. 2). We have extended previous results by explicitly considering random networks, which is a more natural assumption in an economic context.

The numerical results also show that increasing the average size of the neighborhood, i.e. average number of links per agent, $k$, the average fraction of C-agents $f_{C}$ decreases faster with $b$. Several extensions of this model have been studied in the literature: introducing asynchronous updates [HUB 93] or introducing errors in the imitation process [MUK 96], where the basic results shown above persist [NOW 94].

It is also worth noting that the fraction of C-agents depends strongly on the network. As can be seen from Fig. 2, both the regular lattice with first neighbor interaction and the random network with $k=4$ have the same average connectivity, however $f_{C}$ behaves differently with $b$. Same applies for the regular lattice with second neighbor interaction and random network with $k=8$.

In Fig. 3, we plotted the averaged asymptotic value of $f_{C}$ for the fully adaptive network $p=1$ and for different initial networks. The average is over 10 different random initial conditions with a density of $0.6 \mathrm{C}$-agents. Unlike the non-adaptive case, the fraction $f_{C}$ seems to be quite independent of the incentive to defect $b$, and the initial network chosen (regular or random). Only the average connectivity $k$ seems to play a role for determining the asymptotic 

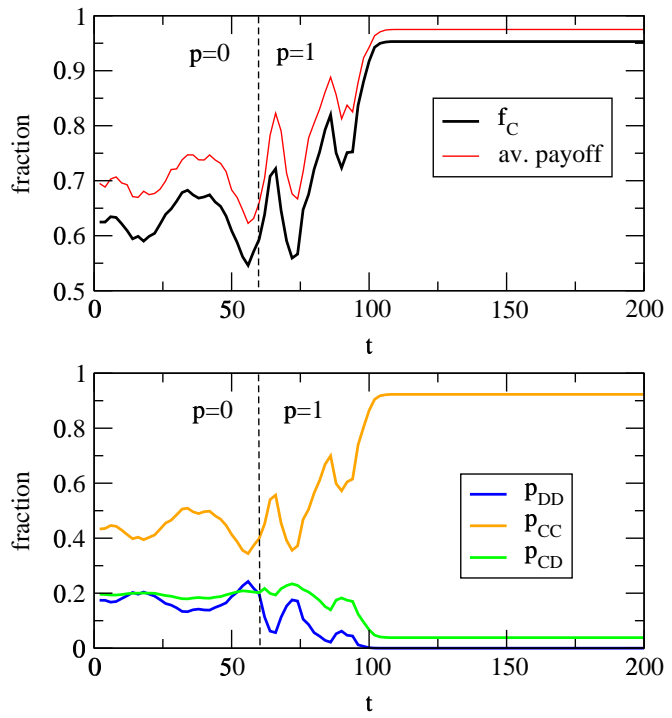

Figure 4: (Top) Time series of C-fraction $f_{C}$ and average payoff in a random network: without network adaptation $(p=0)$ for $t<60$, and thereafter in the full adaptive network $(p=1)$. (Bottom) Corresponding time series of the different link probabilities: two C-agents $\left(p_{C C}\right)$, a $\mathrm{C}$ and a D-agent $\left(p_{C D}\right)$, and two D-agents $\left(p_{D D}\right)$ having a link. Parameter values: $b=1.35, k=8$.

fraction of cooperation. To illustrate how cooperation is enhanced against the non-adapting network, we show in Fig. 4 a simulation where the system first evolves with $p=0$ until at $T=60$ time iterations the adaptation is switched on $(p=1)$. It is clear from the figure that the fraction of C-agents is highly enhanced with respect to the static network.

One of the most interesting features encountered when the network is able to adapt with the game $(p=1)$, is that throughout the whole range of parameters studied $(1<b<2)$, the dynamics settles onto a steady state after some transient time. Figure 4 also shows the time series of the different links probabilities $\left(p_{C C}, p_{C D}, p_{D D}\right)$. The steady state corresponds to a stationary network structure and individual payoffs $\Pi_{i}$. Notice how the network adaptation clearly favors having links between C-agents. Most agents are unsatisfied ${ }^{12}$ in this steady state and they continuously imitate the strategy of their neighbors with highest payoff (all of them C-agents). Also note that at the steady state, the probability of having a link between a $\mathrm{C}$ and a $\mathrm{D}$-agent $p_{C D}$ is finite, while for two D-agents $p_{D D} \rightarrow 0$. This shows that $\mathrm{D}$-agents end up without

${ }^{12}$ In a weak sense, after all they are benefiting from cooperation. 

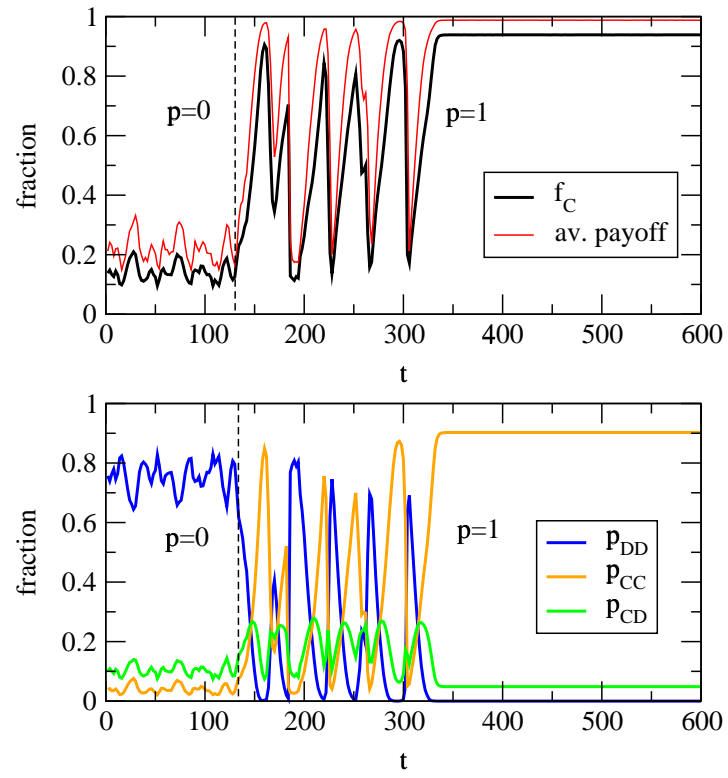

Figure 5: Time series for high $b$. During $0<t<120$, the network is left fixed $(p=0)$, while for $t>120, p=1$. Below the corresponding time series of the link probabilities $p_{C C}, p_{C D}, p_{D D} . b=1.75, k=8$.

links to other D-agents and exploiting other C-agents. Also note that when $p_{D D}=0$, there is no $\mathrm{D}$-agent which will change their neighborhood, so the system may reach a stationary state. In this case D-agents will be satisfied "passive" local maxima, in the sense that nobody is imitating their strategy ${ }^{13}$.

A stationary situation with individual payoffs $\Pi_{i}$ may arise with a number of agents forming, what we shall denote a chain, such that: $s_{l^{m}(i)}=s_{l^{m-1}(i)}=$ $\ldots=s_{l(i)}=s_{i}=1$, where $l^{m}(i)$ is the only agent which is satisfied. All others in the chain are actually unsatisfied, but as they imitate the same strategy they were playing on the previous step, they never change their relative payoff, and the chain becomes a steady state.

An interesting result arises when one looks at the time series for large values of $p(p \sim 1)$ and a high value of the incentive to defect $b$. Before reaching the asymptotic steady state, one observes that large oscillations occur for different choices of initial conditions. Figure 5 shows an example of the dynamics starting from the fixed network. From other initial conditions, similar oscillations are observed. The time evolution of the system before the network is allowed to

\footnotetext{
${ }^{13}$ A highly unlikely situation where two D-agents are neighbors in a steady state would occur if they have exactly the same payoff.
} 


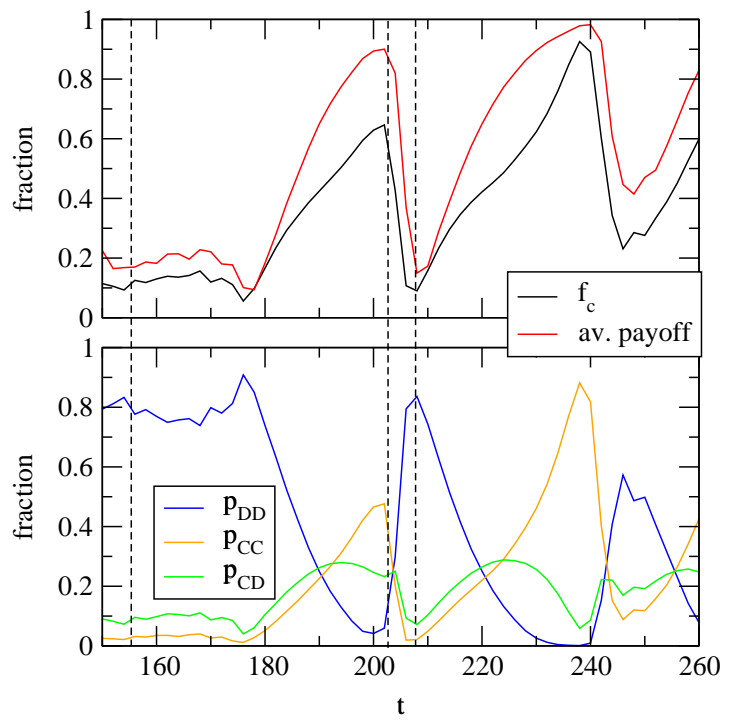

Figure 6: Blow-out of a time series showing avalanches. Parameter values as in Fig. 5.

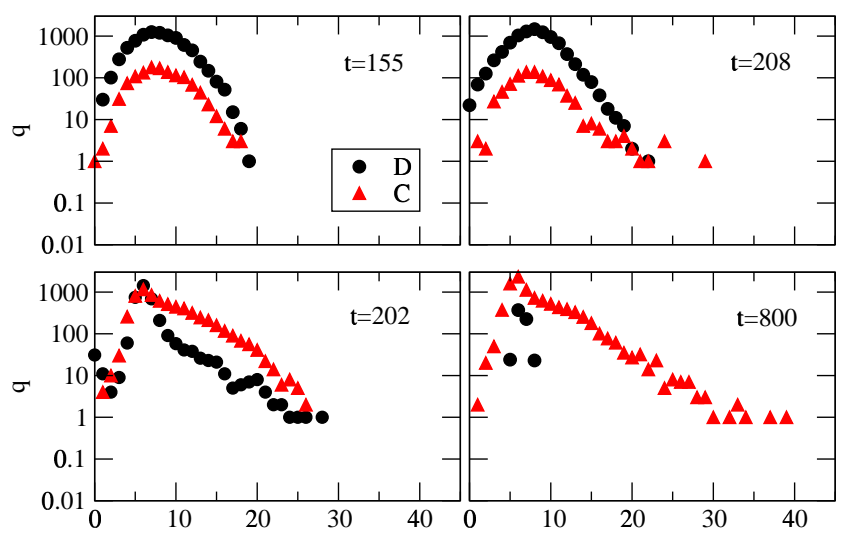

Figure 7: Histogram of number of links, $q$, for a C and D-agents, at different time steps (from time series in Fig. 6) during large oscillations. $t=155$ corresponds to the distributions of the initial random network with $k=8 . t=202$ corresponds to the maximum of $p_{C C}$, while at $t=208$ to the maximum of $p_{D D}$. Finally at $t=800$ the distributions of the asymptotic steady state are shown.

adapt is governed by the high reward of $\mathrm{D}$-agents. However, when the network starts the adaptation $(p=1$ for $t>120)$ the dynamics promotes the creation of 
more links between $\mathrm{C}$-agents, while decreasing dramatically the links between D-agents, as can be seen at time step $t \sim 150$. However, first attempts to build a global cooperative behavior are unsuccessful because the system frustrates. The defecting behavior is so rewarding, that the cooperation has to be built in a specific network configuration in order to be robust against eventual changes of strategy. This correspond to the successive oscillations shown in Fig. 5. The systems reaches a similar fraction of C-agents as in the stationary solution, but several oscillations occur before the stationary regime settles.

In Fig. 6 we show a blow-out of two oscillations. Note the drastic change in the connectivity between $\mathrm{C}$ and $\mathrm{D}$-agents. To quantify this phenomenon we determined the distribution of links for the population of each strategy at different time steps as shown in Fig. 7. The initial distribution $(t=155)$ shows a Gaussian distribution around the starting $k=8$ value. Then at the maximum of $p_{C C}(t=202)$ it is observed that the tails of both distributions extend up to 28 links. Then very rapidly the network switches to the almost defective solution $(t=208)$. However there are a small number of $\mathrm{C}$-agents with a large payoff, which permits the gradual build-up of cooperation. Finally for large times $(t=800)$, the $\mathrm{D}$-agents distribution shrinks to a very narrow distribution, while the $\mathrm{C}$-agents distribution displays a long exponential decay. The stationary network configuration is thus dominated by a few $\mathrm{C}$-agents with a large number of links (the tails of the histogram of links for $\mathrm{C}$-agents). These highly-connected agents dominate the collective behavior of the network.

To illustrate the relevance of the highly connected agents we have built a numerical experiment in which after the system reaches a steady state, the best connected agent, that is the one with a largest number of links switches strategy (from $\mathrm{C}$ to $\mathrm{D}$ ). Figure 8 shows the resulting oscillations, before the system reaches again a (possibly different) steady state. This makes very clear the dominant role of these best connected agents.

\section{Discussion}

The main results of this work are the following. We have introduced a model of cooperation on an adaptive network, where the cooperation is highly enhanced. The network adaptation involves exclusively the D-agents, which in some sense are allowed to "search" for new neighbors, in the hope of finding $\mathrm{C}$-agents to exploit. However our study reveals that this mechanism benefits in the long run cooperators. The asymptotic state reached by the system is a steady state in which the network structure and the average payoff $\Pi$ remain stationary. However, most agents are unsatisfied, and continuously imitate the strategy of their neighbors with highest payoff (most of them C-agents). Also 

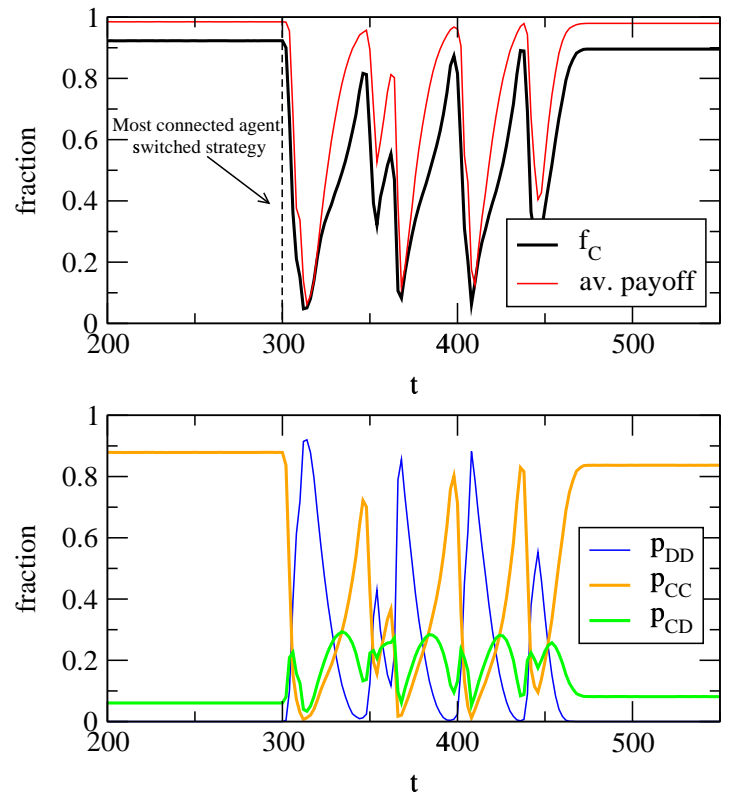

Figure 8: Time series of $f_{C}$, where at $t=300$ the agent with most links changes strategy from $\mathrm{C}$ to D. Parameter values: $b=1.75$ and $k=8$.

the structure of the stationary network presents interesting characteristics. The distribution of links for $\mathrm{C}$-agents has a long exponential tail, with a very few number of highly connected $\mathrm{C}$-agents having up to 4 times the average number of the links in the whole network. These agents dominate the cooperative network structure.

We have also obtained that, for sufficiently high values of the incentive to defect $b$, the induced network structure may suffer large reorganizations. These manifest themselves as large oscillations in the fraction of C-agents, where the network visits for a short time the nearly full cooperative regime, followed by a short time of nearly full defecting regime. In the current dynamical model, these large oscillations are long lived transients, but the system reaches a final stationary state. The interesting aspect is that these large oscillations might be easily triggered by the spontaneous change of the strategy of a highly connected agent.

Acknowledgment. We acknowledge useful discussions with D. Cardona-Coll, P. Battigalli, A. Kirman and J. Weibull. M.G.Z., V.M.E. and M. S. M acknowledge financial support for DGYCIT (Spain) project PB94-1167. 


\section{References}

[AXE 81] R. Axelrod And W. D. Hamilton. The evolution of cooperation. Science, 211:1390-1396, 1981.

[AXE 84] R. Axelrod. The Evolution of Cooperation. Basic Books, New York, 1984.

[BAN 92] A. BANNERJEE. A simple model of herd behaviour. Quarterly Journal of Economics, 108:797-817, 1992.

[COH 99] M. Cohen, R. Riolo And R. Axelrod. The emergence of social organization in the prisoner's dilemma: how context-preservation and other factors promote cooperation. Santa Fe Institute Working Paper 99-01-002, 1999.

[CON 99] R. CONT AND J. P. Bouchaud. Herd behavior and aggregate fluctuations in financial markets. Macroeconomic Dynamics, 1999. In press.

[EGU 99] V. M. Eguiluz and M. G. Zimmermann. Dispersion of rumors and herd behavior. Los Alamos e-print archive (www.lanl.gov): cond-mat/9908069, 1999.

[FER 98] C. Alós Ferrer, A. B. Ania and F. Vega-Redondo. An evolutionary model of market structure. Preprint from http://merlin.fae.ua.es/fvega/\#rp, 1998.

[GOY 99] S. GoYAL AND S. Joshi. Networks of collaboration in oligopoly. Mimeo, 1999.

[HUB 93] B. A. Huberman and N. S. Glance. Evolutionary games and computer simulations. Proc. Natl. Acad. Sci. USA, 90:7716-7718, 1993.

[KIR 93] A. KIRMAN. Ants, rationality and recruitment. Quarterly Journal of Economics, 108:137-156, 1993.

[KIR 99] A. KIRMan. Aggregate activity and economic organisation. Revue Economique des sciences sociales, 113:189-230, 1999.

[LIN 94] K. Lindgren and M. G. Nordahl. Evolutionary dynamics of spatial games. Physica D, 75:292-309, 1994.

[MUK 96] A. Mukherji, V. Rajan and J. R. Slagle. Robustness of cooperation. Nature, 379:125-126, 1996.

[NOW 92] M. A. NOWAK AND R. M. MAY. Evolutionary games and spatial chaos. Nature, 359:826-829, 1992.

[NOW 93] M. A. NowaK AND R. M. MaY. The spatial dilemmas of evolution. Int. Jour. of Bif. and Chaos, 3(1):35-78, 1993.

[NOW 94] M. A. Nowak, S. Bonhoeffer and R. M. May. Spatial games and the maintenance of cooperation. Proc. Natl. Acad. Sci. USA, 91:4877-4881, 1994.

[WEI 96] J. WeIBull. Evolutionary Game Theory. MIT University Press, 1996. 
